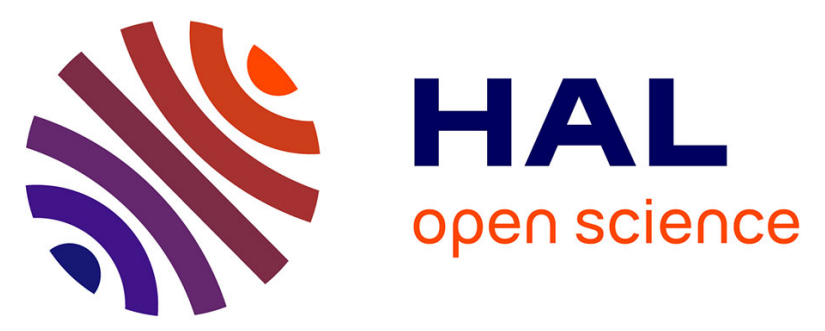

\title{
AlphaRad, a new integrated CMOS System-on-Chip for high efficiency alpha particles counting
}

\author{
D. Husson, A. Bozier, S. Higueret, T.D. Le, A. Nourreddine
}

\section{To cite this version:}

D. Husson, A. Bozier, S. Higueret, T.D. Le, A. Nourreddine. AlphaRad, a new integrated CMOS System-on-Chip for high efficiency alpha particles counting. Nuclear Instruments and Methods in Physics Research Section A: Accelerators, Spectrometers, Detectors and Associated Equipment, 2006, 569, pp.845-852. 10.1016/j.nima.2006.09.110 . in2p3-00127085

\section{HAL Id: in2p3-00127085 \\ https://hal.in2p3.fr/in2p3-00127085}

Submitted on 29 Jan 2007

HAL is a multi-disciplinary open access archive for the deposit and dissemination of scientific research documents, whether they are published or not. The documents may come from teaching and research institutions in France or abroad, or from public or private research centers.
L'archive ouverte pluridisciplinaire HAL, est destinée au dépôt et à la diffusion de documents scientifiques de niveau recherche, publiés ou non, émanant des établissements d'enseignement et de recherche français ou étrangers, des laboratoires publics ou privés. 


\title{
AlphaRad, a new integrated CMOS System-On-Chip for high efficiency alpha particle counting.
}

\author{
D.Husson $^{* 1}$, A.Bozier ${ }^{2}$, S.Higueret ${ }^{3}$, T.D.Le ${ }^{3}$, A.Nourreddine ${ }^{1}$ \\ ${ }^{1}$ Université Louis Pasteur and IPHC (UMR7178), 23 Rue du Loess, BP 28, F- 67037 \\ Strasbourg Cedex 2, France \\ ${ }^{2}$ InESS (UMR7163) F- 67037 Strasbourg Cedex 2, France \\ ${ }^{3}$ IPHC (UMR7178) 23 Rue du Loess, BP 28, F- 67037 Strasbourg Cedex 2, France
}

\begin{abstract}
An integrated System-on-Chip (SoC) has been designed in 0.6 $\mu \mathrm{m}$ CMOS mixed analog/digital technology, and tested for high rate alpha particle counting. The sensor is the most innovative part of the chip, with a total active area of two times $2.5 \mathrm{~mm} \times 5 \mathrm{~mm}$. The two-stage charge-to-voltage amplification scheme includes a numerical block for offset compensation. The chip has been tested in alpha sources: a very high signal over noise ratio was obtained, leading to a detection efficiency of $5 \mathrm{MeV}$ alpha particles close to $100 \%$. The chip is working at room temperature and has been tested up to 300 $\mathrm{kHz}$ reset frequency.
\end{abstract}

Future applications of this SoC will focus on detection of fast and thermal neutrons free of gamma contamination.

PACS: 29.40.Wk; 07.50.-e; 07.77.Ka; 28.41.Rc;

\footnotetext{
* corresponding author : daniel.husson@ires.in2p3.fr
} 
Key words: Solid state detectors; System-on-chip; Alpha particles; Neutrons.

The field of dosimetry is rapidly changing under stronger societal demands for radioprotection and new legal standards imposed by the European Commission [1]. Passive and operationnal dosimetry systems are presently available for a wide range of radiation types and energies, but efficient detection of neutrons (free of gamma contamination) is still demanded, as well as flexible, efficient and cheap systems for gaseous radon monitoring [2]. The present development adresses both problems, as an efficient and miniaturized system for alpha particle detection is able to detect neutrons, through $(n, \alpha)$ reactions at low energies or recoil protons at the $\mathrm{MeV}$ scale.

Barrier-surface diodes are commonly used for alpha detection, but the high capacitance of large sensitive areas prevents the integration of such a device in VLSI circuits. The solution for integration of a large detecting surface of low capacitance and low leakage current was suggested to us by CMOS pixel sensors, which proved high performance for tracking of low ionising particles (MIPs). Integrated circuits in CMOS technology provide high flexibility at low price, as they use standard industrial silicon. In addition to this, our chip offers the nice feature of being naturally transparent to gamma radiation.

The paper is organised as follows: the first part is devoted to the description of the chip, with special emphasis on the sensor. In the second part, 
we present the response of the chip to various tests, electrical behaviour and response to alpha sources as well as pulsed blue light.

\section{The AlphaRad chip}

Application of CMOS Active Pixel Sensors (APS) to single charged particle detection [3] is under fast development, with tracking of Minimum Ionising Particles (MIPs) already at the level of one micron $\sigma_{\mathrm{xy}}$ resolution. A well known drawback of any CMOS system is the poor energy resolution it offers, but applications like vertexing (tracking of MIPs) do not require energy measurement. The lack of energy measurement in CMOS pixels is inherent to their design, because of two specific features: (i) the intrinsic thinness of the sensor makes that only a fraction of the charge signal is sampled, and (ii) the excess carriers generated in silicon are collected by pure diffusion, as the sensor has no depleting electric field. In the case of alpha particles as well, many applications do not need energy measurement, and our SoC demonstrates that fast counting on a large surface can be achieved without pixellisation. The AlphaRad chip has no individual pixel readout, and offers instead a single output and full integration. Other core advantages of our device are high flexibility of the electronic functions to be implemented, room temperature operation, low leakage current, high efficiency to alphas and $\mathrm{MeV}$-protons, high counting speed, and natural transparency to gammas, all features that are most promising for neutrons detection. 
The first experimental detection of alphas from ${ }^{222} \mathrm{Rn}$ by a CMOS chip with active pixels has been reported elsewhere [4]. This experiment was performed with a sophisticated acquisition system requiring megabtytes of memory per event, correlated double-sampling and heavy off-line treatment to reconstruct the tracks: to illustrate this, a typical alpha particle in the MIMOSAV chip generates a cluster of 100 pixels [4] and the data on most of the one million pixels (doubly sampled) has to be suppressed. We present here a dedicated chip for efficient and fast $\alpha$ counting without any post-processing.

The AlphaRad chip (Fig1) has two identical and independent detection chains, each one including a 2.5 x $5 \mathrm{~mm}$ sensor driven by a large reset transistor, followed by a double amplification stage (with an numerical block for offset-compensation on the second amplifier) and a final binary comparator output. Keeping in mind the applications to dosimetry, we duplicated the sensor+chain in two independent matrices, each one being able to deliver separate signals with the adequate converter (e.g. for neutrons: $\mathrm{n} \rightarrow \alpha$ or $\mathrm{n} \rightarrow \mathrm{p}$ ) .

As the amplification chain of this first prototype is quite conventional, the paper will focus on a detailed description of the sensor.

\section{1) The sensor}

It is now demonstrated [3] that charge collection at the fC level can be achieved on standard commercial silicon without depletion zone, therefore no 
need for high resistivity silicon neither high electric fields. In good quality silicon (e.g. epitaxial), excess carrier life-times longer than $100 \mathrm{~ns}$ are currently achieved, and thermal diffusion without electric field ensures efficient charge collection on micron-size diodes much smaller than the active sensitive volume. In a true active pixel system for low ionising particles [3], the typical distance between diodes is $d_{i d}=20 \mu \mathrm{m}$.

For alpha particles in the $\mathrm{MeV}$ range, the charge signal in silicon is thousand times the one of relativistic particles (minimum ionising). This larger linear energy transfer (LET) allows to push the concept of collection by diffusion a step further. A rough calculation with a diffusion constant of $D_{e}=0.004 \mathrm{~m}^{2} \mathrm{~s}^{-1}$ gives a diffusion length $\mathrm{L}=(\mathrm{D} . \tau)^{1 / 2}$ of about $2 \mu \mathrm{m}$ per $n s$ of lifetime (at room temperature), and currently available silicon offers minority carriers life-times up to one microsecond. Detailed simulation studies [5] have demonstrated that, even with conservative life-times $\left(\tau_{\mathrm{e}}>200 \mathrm{~ns}\right)$, a pure diffusion process in a $15 \mu \mathrm{m}$ deep epitaxial layer leads to a charge collection higher than $75 \%$ for inter-diode distances as large as $\mathrm{d}_{\mathrm{id}}=80 \mu \mathrm{m}$.

The long diffusion length $\mathrm{L}_{\mathrm{e}}$ of excess electrons is the key parameter of the AMS 0.6 technology with epi layer, allowing to design a large sensitive area (large at the scale of integrated VLSI circuits), with a single readout for the whole surface and low figures for noise (leakage current and capacitance). For the AMS design of $\mathrm{n}^{+} / \mathrm{n}$-well/p diodes, the junction is precisely located at the 
interface between the larger n-well and the p-type Si. The capacitance of a 3x3 $\mu \mathrm{m}$ n-well diode (5V inverse biased) has been measured and calculated to be of $2.5 \mathrm{fF}$ [6]: this allows to connect several hundreds of diodes in parallel with still a limited capacitance. Considering now the other major source of electronic noise in silicon diodes - shot noise from the leakage current - the argument works the same way: with a current density of $0.01 \mathrm{fA} / \mu \mathrm{m}^{2}$, segmentation of a large junction of $\mathrm{cm}^{2}$ size into a finite number of small diodes allows to benefit from a large area with moderate currents.

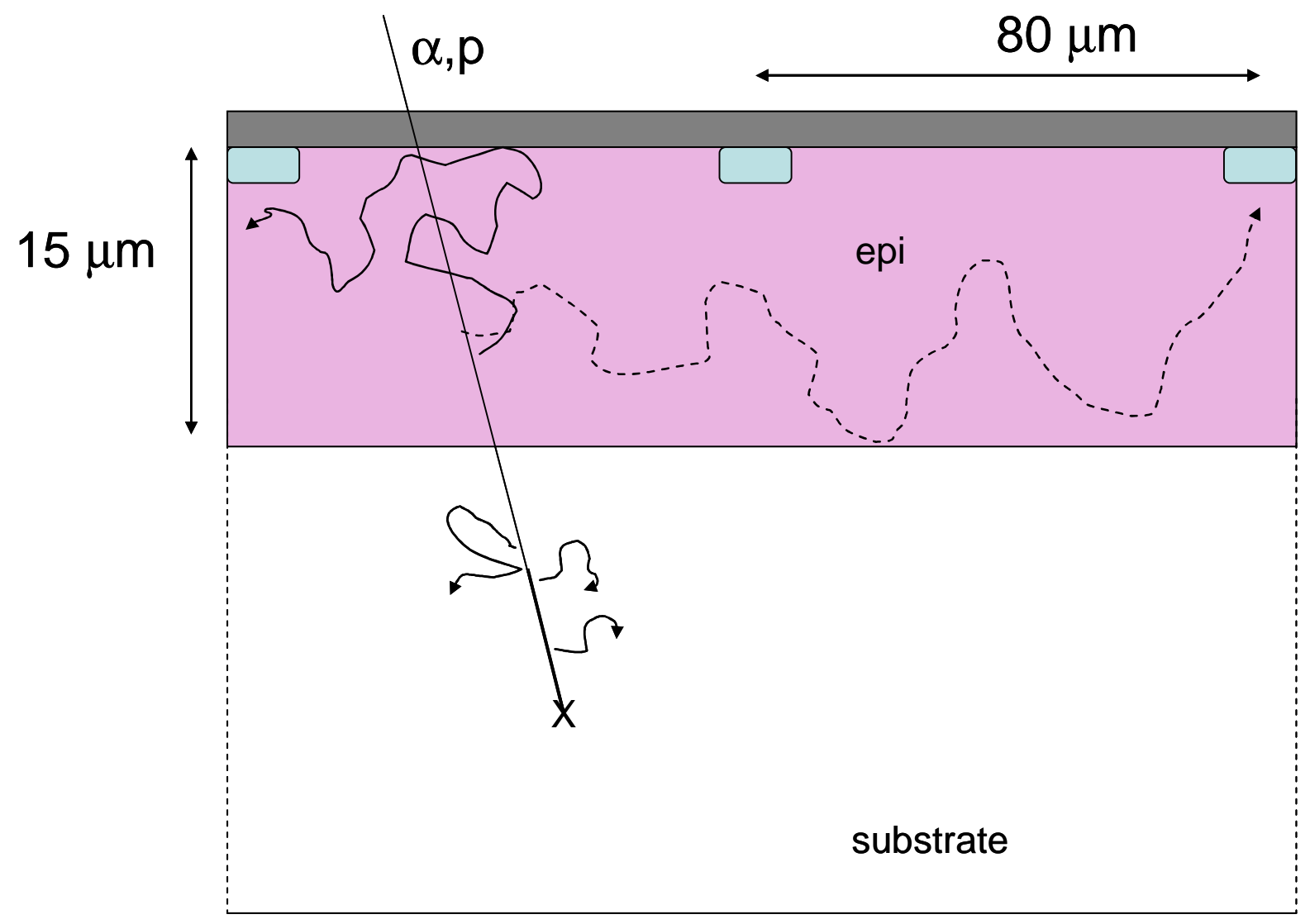

Fig 1: Detecting principle and key dimensions of the sensor part: the small $\mathbf{n}+/ \mathbf{n} / \mathbf{p}$ diodes are 80 micrometer apart . 
The detailed concept of the AlphaRad sensor is now the following (Fig1): the sensitive volume is a $15 \mu \mathrm{m}$ deep epitaxial layer of semi-resistive silicon $\left(\mathrm{N}_{\mathrm{A}}=10^{15} \mathrm{~cm}^{-3}\right)$; it is limited on the top by the oxide layer, on the bottom by the substrate, made of lower quality silicon; the excess charges created in the epilayer are collected by thermal diffusion on small $\mathrm{n}^{+} \mathrm{np}$ diodes. Each diode is of $3 \times 3 \mu \mathrm{m}$ size, and the complete collection happens in less than $1 \mu \mathrm{s}$. Thanks to the built-in potential at the interface $\mathrm{p}$-epi/p-substrate, the excess carriers created in the epi layer do not escape to the substrate during diffusion. The diodes have no active pixel adressing system, they are just connected in parallel through standard aluminum lines running on the oxide layer. In the AMS 0.6 technology, the geometry of the grid (Fig2) could be optimized for an interdiode distance of $\mathrm{d}_{\mathrm{id}}=80 \mu \mathrm{m}$, and one full array of $32 \times 64$ diodes covers a total sensitive area of $2.56 \mathrm{~mm} \times 5.12 \mathrm{~mm}$. The signal is handled by a single chargeto-voltage amplifying chain outside the sensitive area.

The noise calculation is given hereafter with some details. A single diode of dimensions WxL behaves like a capacitor $\mathrm{C}_{\mathrm{d}}$ which is the sum of two contributions, $C_{j}$ (surface) and $C_{j s w}$ (perimeter), with the values $C_{j}=0.084 \mathrm{fF} / \mu \mathrm{m}^{2}$ and $\mathrm{C}_{\mathrm{jsw}}=0.51 \mathrm{fF} / \mu \mathrm{m}$ for the AMS 0.6 process. The total capacitance is therefore $\mathrm{C}_{\mathrm{d}}=\mathrm{WLC}_{\mathrm{j}} /(1+\mathrm{x})^{\mathrm{m}}+2 \cdot(\mathrm{W}+\mathrm{L}) \mathrm{C}_{\mathrm{jsw}} /(1+\mathrm{x})^{\mathrm{m}}{ }^{\prime}$ where $\mathrm{x}=\mathrm{V} / \mathrm{V}_{\mathrm{b}}$ (the exponants $\mathrm{m}=0.41$ and $\mathrm{m}^{\prime}=0.31$ are given by the manufacturer). For a grid structure of $\mathrm{N}$ diodes, we have $C_{\text {tot }}=N \cdot C_{d}+C_{\text {grid }}$, where the additional term is typically $10 \mathrm{fF}$ for $80 \mu \mathrm{m}$ 
of aluminum (the metal width is $0.9 \mu \mathrm{m}$ for metal 1,2 and $1.1 \mu \mathrm{m}$ for metal 3 ). Finally, for this design $\left(\mathrm{N}=32 \times 64, \mathrm{~d}_{\mathrm{id}}=80 \mu \mathrm{m}\right)$ and the parameters of this technology, the total capacitance of the sensor is $\mathrm{C}_{\mathrm{d}}=40.9 \mathrm{pF}$. This value is by no means negligible at the VLSI scale, and it puts severe constraints on the reset transistor and on the noise figure of the whole chain.

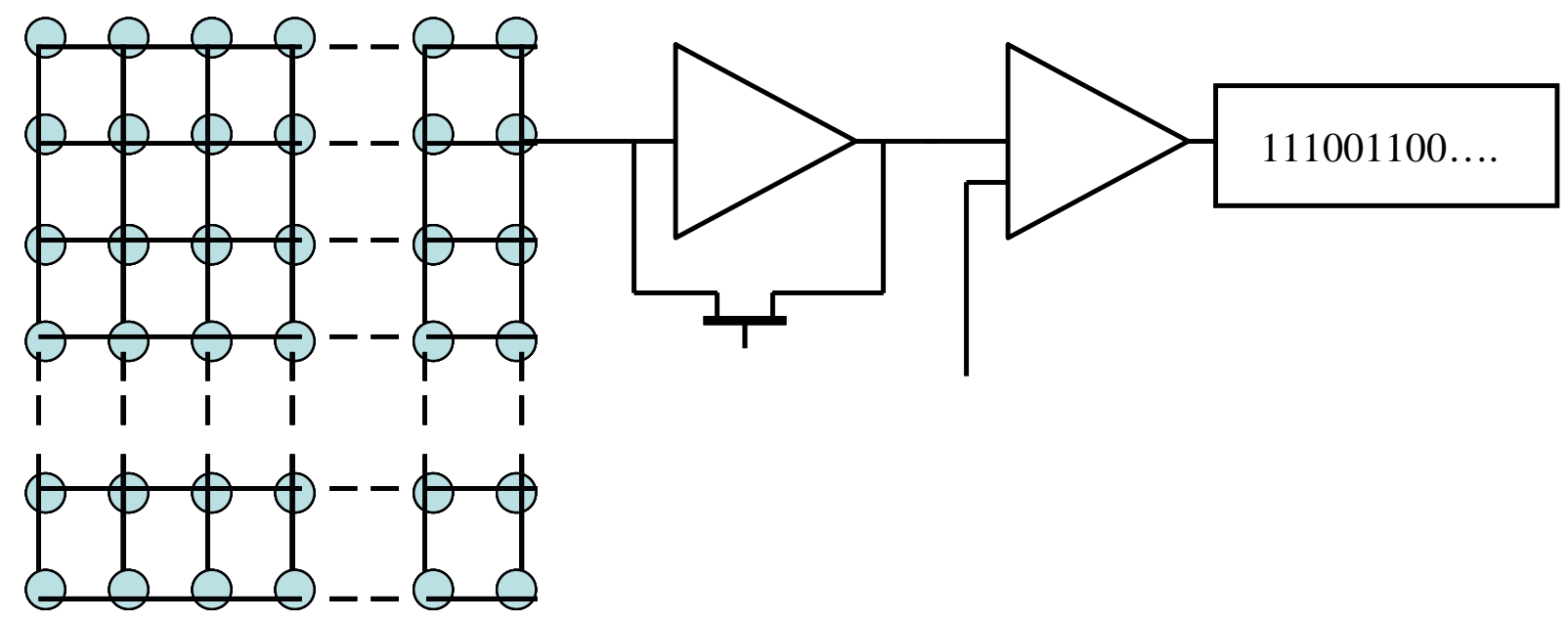

Fig 2: Functional sketch of the sensor (32x64 passive diodes in parallel) with a single electronic treatment made of three blocks: amplification, discrimination and counting.

A single $5 \mathrm{MeV}$ alpha particle generates a detectable charge of $\mathrm{Q}_{\alpha}=64 \mathrm{fC}$ (400000 electrons) hence a voltage drop of $\Delta \mathrm{S}_{\mathrm{in}}=1.56 \mathrm{mV}$ on a $41 \mathrm{pF}$ capacitor. A voltage gain of 700 is needed to get an output signal of $\Delta S_{\text {out }}=1 \mathrm{~V}$. For reasons explained below, amplification is performed in a two stage scheme: a first 
amplifier of gain 70 is followed by a second one of gain 10, this second stage being used to implement the numerical block for offset compensation (Fig3).

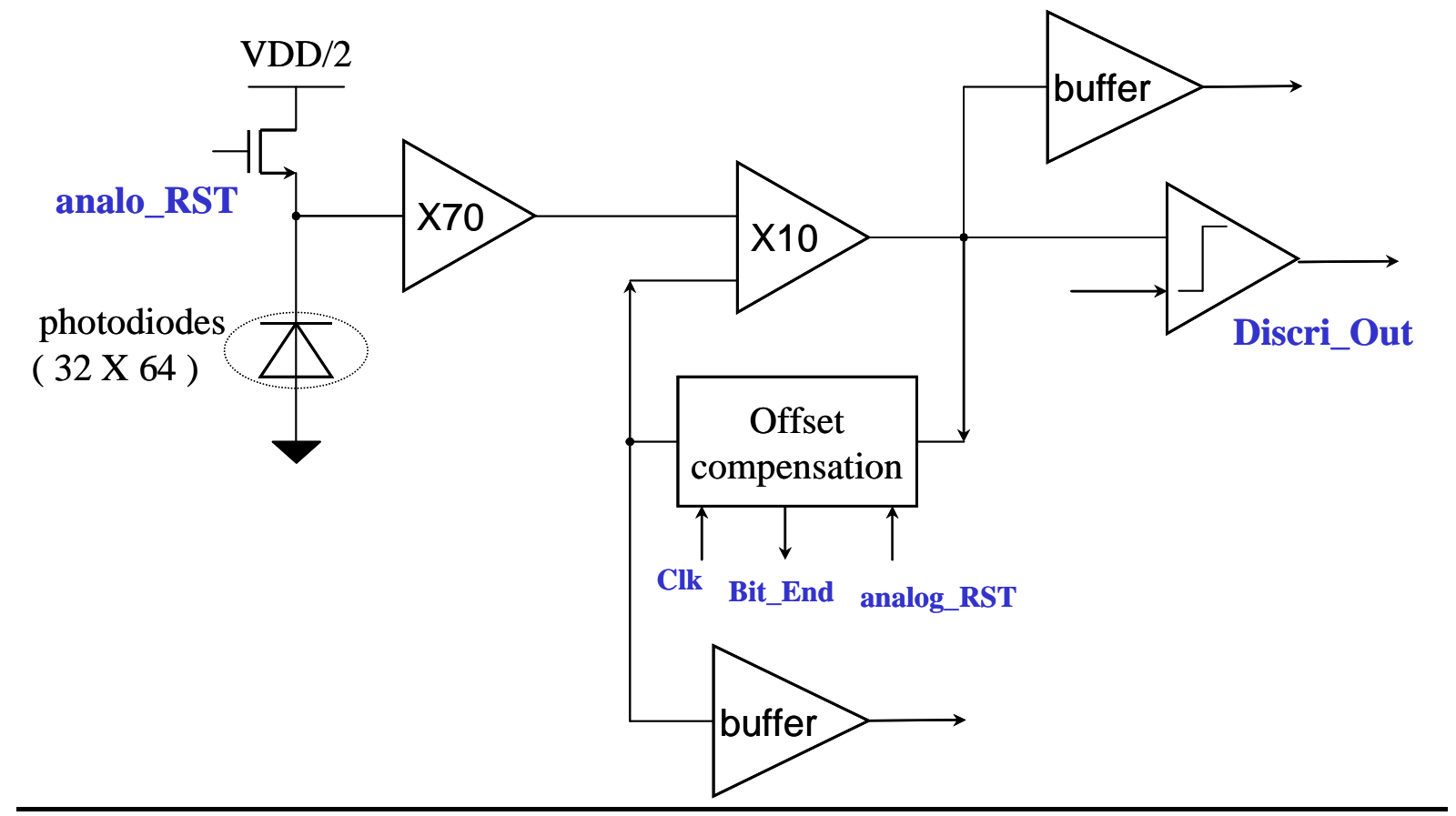

Fig 3: Schematic of the amplification chain

\section{2) The reset transistor}

The diodes are slightly inverse-polarised $\left(\mathrm{V}_{\mathrm{DD}}=5 \mathrm{~V}\right)$, and the tiny depleted zone around the $\mathrm{N}$-well is less than $0.5 \mu \mathrm{m}$ thick, with a leakage current density of typically $\mathrm{J}_{\mathrm{s}}=10^{-17} \mathrm{~A} / \mu \mathrm{m}^{2}$. A large area diode of the size of one full matrix $\left(12.5 \mathrm{~mm}^{2}\right)$ would generate a dark current of several $\mathrm{nA}$, but with our choice of diode segmentation, the total leakage current of the sensor is only $\mathrm{I}_{\text {leak }}=5.1 \mathrm{pA}$, say three orders of magnitude lower. This current, even small, is continuously flowing to the sampling transistor which has to be reset periodically. 
The RST signal is generated through a reset transistor $\mathrm{T}_{\mathrm{RST}}$ (Fig3) which must be designed carefully. At each RST cycle, the $\mathrm{T}_{\mathrm{RST}}$ (of NMOS type) is connected in series with the sensor $\mathrm{C}_{\mathrm{d}}$. Choosing to keep the RST time below 80 ns puts severe constraints on the $\mathrm{W} / \mathrm{L}$ geometry of the $\mathrm{T}_{\mathrm{RST}}$. In effect, the thermal noise should be low and, first of all, the peak current during RST must be kept under control to avoid destruction of the transistor. The optimal parameters of the $\mathrm{T}_{\mathrm{RST}}$ are the following: $\mathrm{W} / \mathrm{L}=20 / 0.6 \mu \mathrm{m}$ for a switching time of $70 \mathrm{~ns}$ and a peak source current limited to $9.5 \mathrm{~mA}$. The RST time is to be considered as dead time for the detector.

\section{3) The amplification and offset-compensating block}

The ionising power of a $5 \mathrm{MeV}$ alpha particle penetrating a $2 \mathrm{~g} / \mathrm{cm}^{3}$ material is $140 \mathrm{keV} / \mu \mathrm{m}$, according to the Bethe-Bloch model. At this energy, the full range of the incoming alpha is $24 \mu \mathrm{m}$ of $\mathrm{Si}$ [7]. In our device and for normal incident tracks, the Bragg peak is located deep inside the substrate (Fig1), where carriers dynamics is dominated by fast recombination. Long diffusion times are to be found only in the epitaxial layer, which is the true sensitive zone, and we integrate Bethe-Bloch only in this region, considering that the remaining energy is lost. This gives our central value $Q_{\alpha}=400000$ electrons. 
With the five key parameters fixed so far (signal magnitude, $\mathrm{C}_{\mathrm{d}}$ and $\mathrm{I}_{\text {leak }}$ of the sensor, W/L of the RST transistor), a rough calculation of noise gives a minimal RMS voltage of $\mathrm{e}_{\text {sensor }}=10 \mu \mathrm{V}$ before any contribution of the amplifying chain. Obviously, a low-noise amplifier is not really useful, and we decided for convenience to use a standard block (Miller-OTA amplifiers) which can be run to $\mathrm{MHz}$ frequencies, with a mid-point voltage fixed to $\mathrm{V}_{\mathrm{DD}} / 2=2.5 \mathrm{~V}$, and a typical voltage excursion of $\Delta \mathrm{V}_{\mathrm{in}}=1.56 \mathrm{mV}$ for a single $\alpha$ particle. For this first prototype, we chosed to use the same OTA basic block several times in the different stages (amplification, offset compensation and comparator).

A well known drawback of operational amplifiers is the slow drift in time of the input voltage, and this offset has to be compensated periodically. Our choice was to amplify a first time with a gain of $\mathrm{g}_{1}=70$, therefore $\Delta \mathrm{V}_{\text {out_1 }}=112$ $\mathrm{mV}$ as input voltage excursion of the second OTA. This second amplifier $\left(\mathrm{g}_{2}=10\right)$ is the one who needs the highest common mode rejection (CMR), which was fixed at $0.5 \mathrm{~V}$ as a conservative value. At this node, the offset compensation is performed at $1 \mathrm{MHz}$ frequency, delivering an End_Bit after 8 $\mu$ s when compensation is completed. This operator is quite standard and doesn't need much development. The functioning of the chip during this phase can be seen on the oscilloscope display (Fig4). The total gain-band-width product of the chain GBW is calculated to be $16 \mathrm{MHz}$. 
To summarize the sequential functioning of the chip, the RST signal is delivered at a typical frequency of $10 \mathrm{kHz}$, and in this interval, several $\alpha$ particles (up to 3) can be detected before saturation. The compensation (on 8 bits at $1 \mathrm{MHz}$ ) is performed in much longer intervals (typically $t_{\text {comp }} \sim 1 \mathrm{~s}$ ). These additional $8 \mu$ s are another source of dead time of the system, but $t_{\text {comp }}$ can be set to longer values if needed.

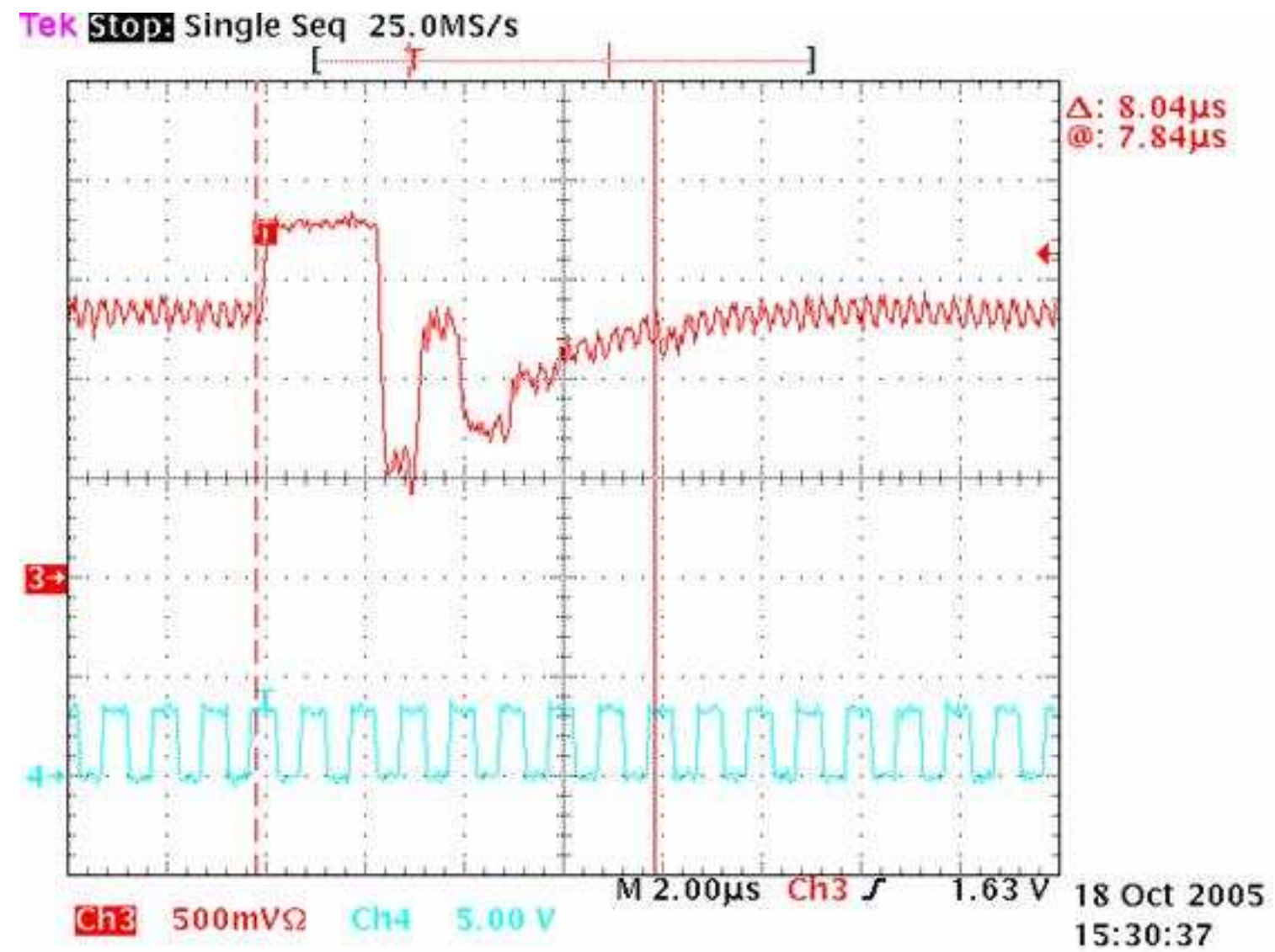

Fig 4: Oscillogram of the chip during the offset compensation stage:

the 8-bits DAC works at $1 \mathrm{MHz}$ and resets the mid-point voltage to $2.5 \mathrm{~V}$.

A photograph of the complete layout of the AlphaRad may be seen on Fig5, which shows that the sensor part occupies most of the silicon surface. The 
two sensitive areas are completely independent. The two amplification chains and output pads are visible on both sides.

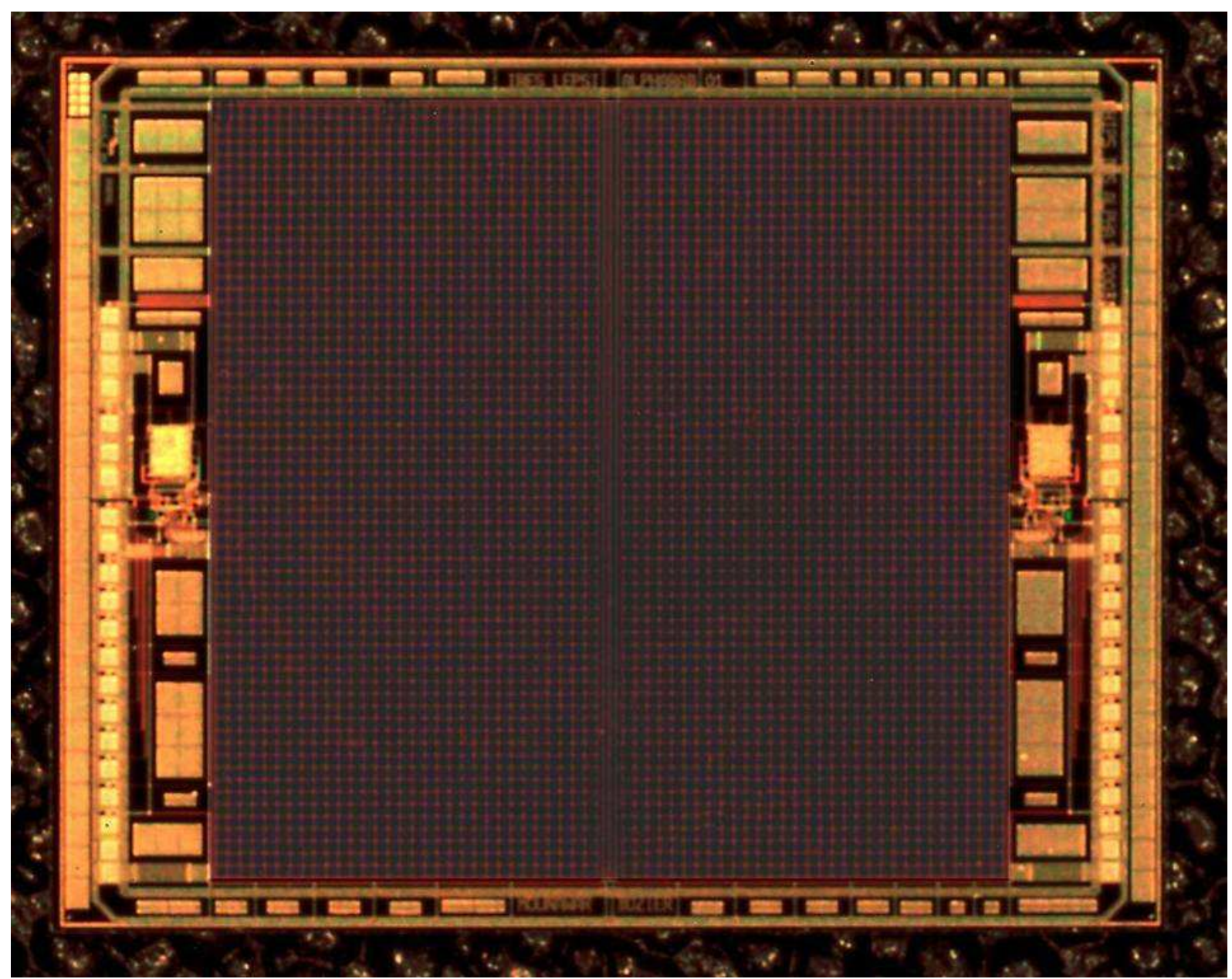

Fig 5: The AlphaRad chip with its two identical sensors. On both sides, the mixed analog-digital amplification chains and output pads. 


\section{Tests of the chip}

The AlphaRad chip has been extensively tested, first at the electrical level to assess the noise performance and thereafter in alpha sources for efficiency and also under pulsed light excitation to check the maximum detection frequency. We give also the noise calculation before comparison to the measured value.

\section{1) Noise of the full chain}

As stressed above, most of the electronic noise is generated in the sensor itself, with a dominant kTC contribution due to the sensor capacitance driven by a large reset transistor. The classical model for noise calculation is a current source $I_{\text {leak }}$ in parallel with the $C_{d}$ capacitor, both connected in series with the $R_{o n}$ resistor (when $T_{R S T}$ is open). This resistor generates (i) a thermal noise (filtered by the $\mathrm{R}_{\mathrm{on}} \mathrm{C}_{\mathrm{d}}$ ) which is simply given by $\overline{V^{2}{ }_{k T C}}=\frac{k T}{C}$. Numerically, the kTC contribution is of RMS $=10 \mu \mathrm{V}$. The second main source of noise is (ii) the shot noise, obtained by integration of the leakage current of the detector equivalent diode: $\overline{V^{2} \text { int }}=\frac{q I_{\text {leak }}}{C^{2}} t$. Even with an integration time as long as $\mathrm{t}=1 \mu \mathrm{s}$, the shot noise is calculated to be of less than $0.1 \mu \mathrm{V}$ RMS. Clearly the shot noise remains well below the dominant kTC contribution, and the chip is to be operated safely at room temperature. 
The contribution of the amplification chain is straightforward as we have to add up in quadrature the R-2R filter of the compensation block $\left(\overline{e^{2}}=4 k T R\right)$ and the two OTA amplifiers, with integration on the full frequency band. The integration range $\Delta \mathrm{f}$ was taken to be $[0.1 \mathrm{~Hz} ; 250 \mathrm{kHz}]$. The lower limit is very conservative as the compensation cycle is rather of $t_{\text {comp }} \sim 1 s$. The noise contribution of the first OTA (of highest gain) dominates the second stage, and with a good approximation, the Equivalent Noise Charge at the input may be expressed as the simple sum of the dominant contributions (sensor plus noisyest OTA) $E N C=\sqrt{\frac{k T}{C}+\overline{e^{2} \text { OTA70 }}}$, where $\overline{e_{\text {OTA70 }}^{2}}=\overline{e^{2} \text { OTA }}+4 k T R_{1} . \Delta f$ (we have $\mathrm{R}_{1}=1 \mathrm{k} \Omega$ for the first OTA). The total RMS noise voltage of the whole chain including the sensor is calculated to be of $25 \mu \mathrm{V}$ at the input. After a voltage gain of 700 , the noise design figure is of less than $20 \mathrm{mV}$, and this should allow to detect highly ionising alpha particles with good efficiency.

Measurement of the electronic noise of the chip was performed as follows. The signal fluctuations are to be observed directly on the oscilloscope, but this kind of measurement is affected by sudden fluctuations of the baseline voltage, and it includes the noise of the additional output buffer. The intrinsic noise is better measured by the classical indirect method, testing the counting rate in the dark for different settings of the comparator level, from a maximum where no count is registered until the minimum where the electronic noise triggers the discriminator continuously. This typical "sigmoid" curve is the 
integral of the noise. By differentiation, one obtains the gaussian shape shown in Fig6. Measured this way in the dark, we get a total RMS voltage noise of 16 $\mathrm{mV}$ at the output, which is very close to the foreseen value.

The standard presentation of noise performance is to compare the noise voltage to the signal voltage for the typical particle to detect: for $5 \mathrm{MeV}$ alpha particles, the chip has a comfortable signal over noise ratio of more than 90 .

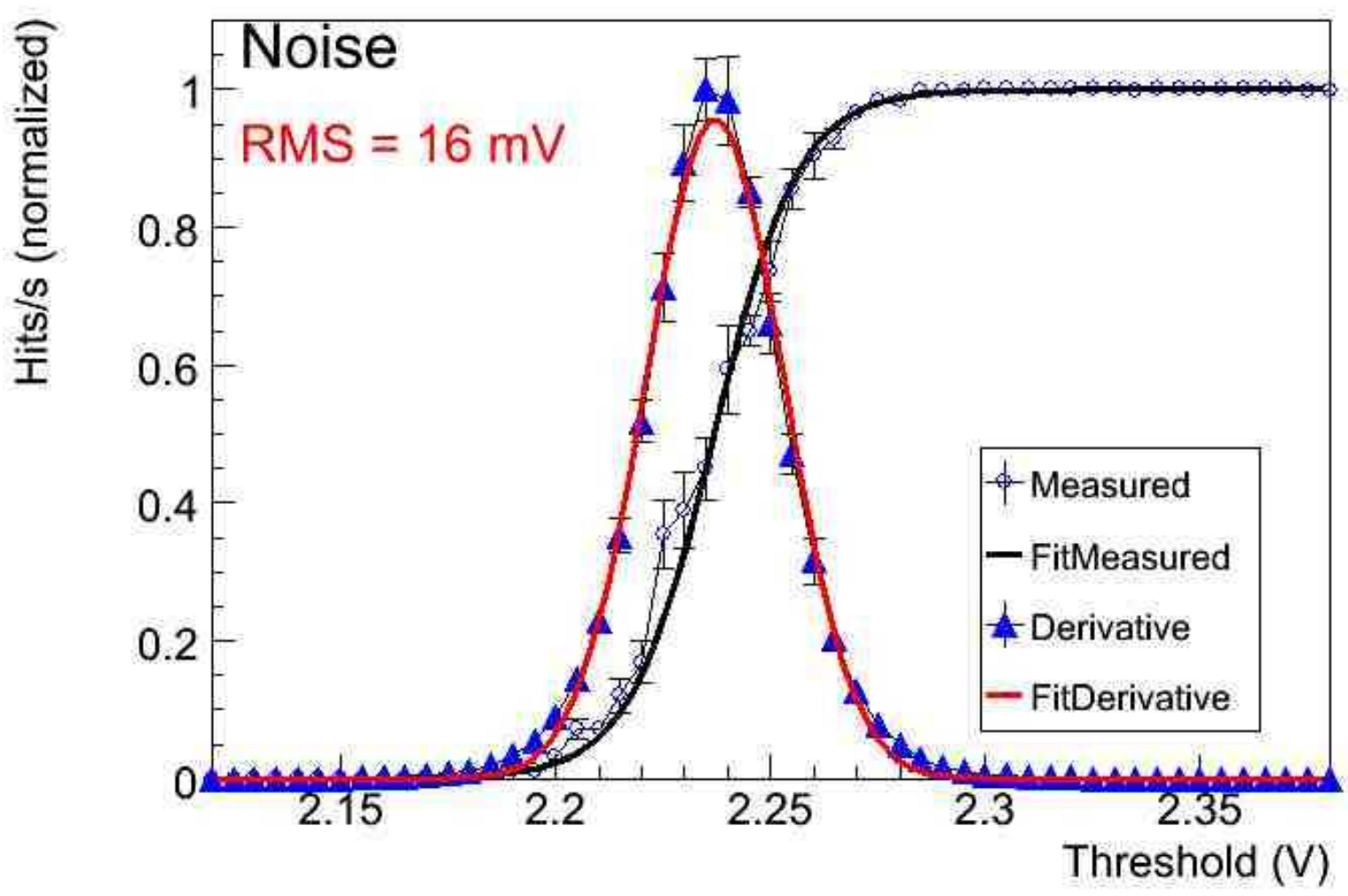

Fig 6: Electronic noise of the AlphaRad chip measured in the dark as a function of the threshold voltage of the discriminator. 


\section{2) Efficiency in alpha source}

The chip has been tested for alphas in a ${ }^{241} \mathrm{Am}$ source $\left(\mathrm{E}_{\alpha}=5.5 \mathrm{MeV}\right)$. The source was conditioned as a quasi-disk of radius $r_{s} \sim 3 \mathrm{~mm}$, protected by a submicrometer thin protection film and deposited on aluminium. The source activity, measured with a calibrated scintillator, was of $26500 \mathrm{~Bq}$ in the full space $(4 \pi)$. The gamma lines of the source are completely invisible, with either a very long absorption length (for $\mathrm{E}_{\gamma}>20 \mathrm{keV}$ ) compared to the epi thickness, or, for the lowest energies $\left(\mathrm{E}_{\gamma}<20 \mathrm{keV}\right)$, remaining below the discriminator level set at five standard deviations of noise.

For these tests, the chip was kept at fixed position and the source was mounted on a mechanical $x-y$ table with a vertical support to vary the distance $\mathrm{z}=\mathrm{d}_{\mathrm{sc}}$ between source and chip. Without vacuum, $\mathrm{d}_{\mathrm{sc}}$ has to be lower than the range of $5.5 \mathrm{MeV}$ alphas in air $(40 \mathrm{~mm})$, but in practice, the maximal distance is even smaller than $20 \mathrm{~mm}$, as a $5 \mathrm{MeV}$ alpha particle releases about $1 \mathrm{MeV}$ in the $5 \mu \mathrm{m}$ thick silicon oxide covering the chip [7]. We didn't perform the test in vacuum, keeping in mind a possible application to radon in air [4], and the test bench was just kept at atmospheric pressure in the dark. A typical result is displayed in Fig7 (error bars are purely statistic). 


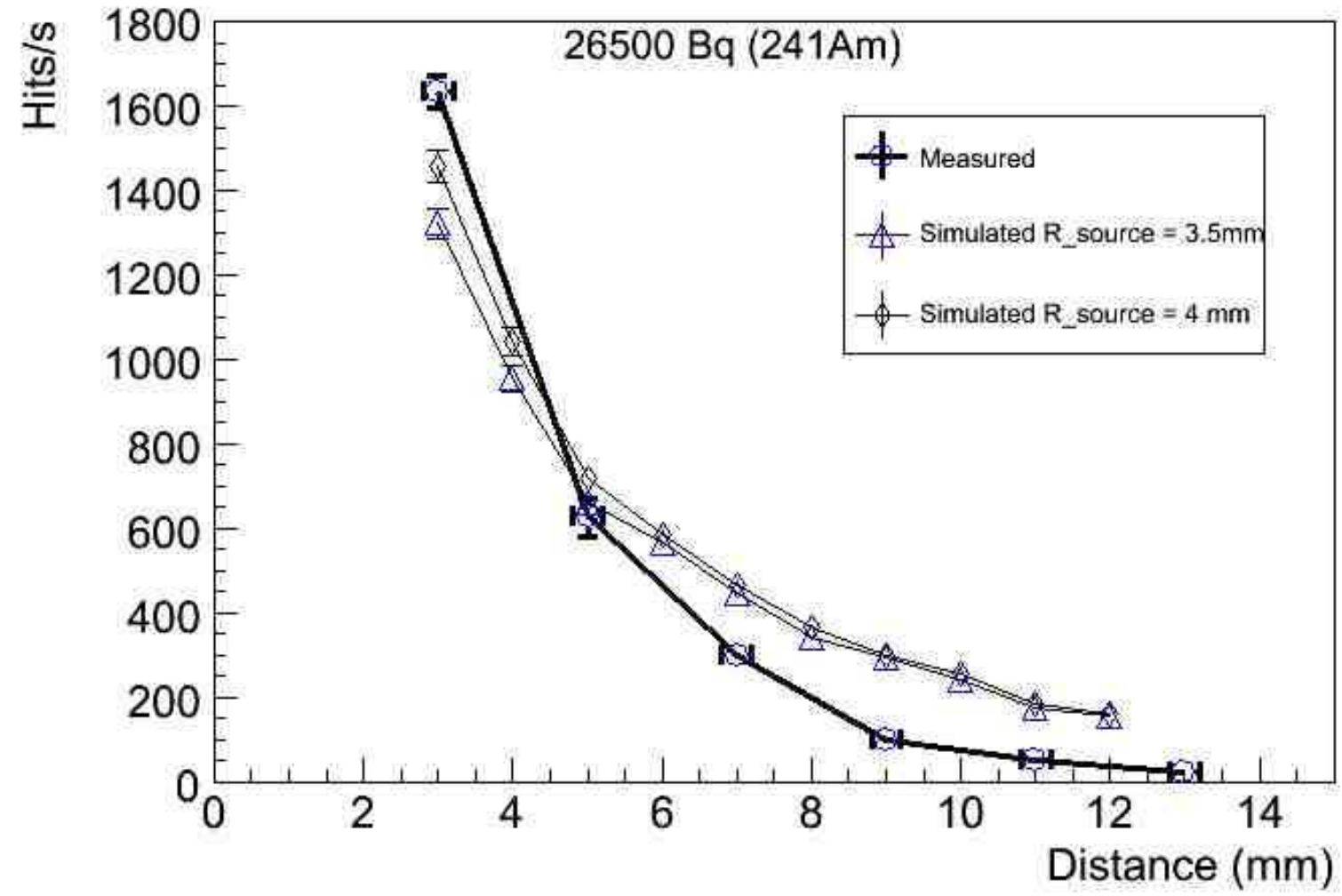

Fig 7: Data versus simulation: counting rate of the AlphaRad in a calibrated $\alpha$-source as a function of chip-to-source distance.

For a geometry like this one, with a source of size D, a sensor D' and distances $d$ all of the same order (d D D'), the counting rate is by no means a function of $1 / \mathrm{d}^{2}$. In order to determine an absolute efficiency, our data were compared to a Monte-Carlo simulation, to take into account geometrical effects as well as absorption in air and in the 5 microns silicon oxide at all angles. The simulation allows to include the small uncertainity on the true size of the source (for $r_{s}=3.5 \mathrm{~mm}$ and $4 \mathrm{~mm}$ on Fig7), a purely geometrical effect which is clearly visible at short distances. Comparison of data to the Monte-Carlo calculation indicates that the absolute efficiency for $\alpha$ detection is very close to $100 \%$, as 
could be expected from the very high signal to noise ratio. The small discreapancies are probably source effects, lack of uniformity on one hand, and also autoabsorption (small energy losses) which lowers the counting at large distances.

\section{3) Test in pulsed blue light}

For a system without external trigger like this one, the random nature of radioactive sources prevents to predict the arrival time of any particle. In order to control the time delay between detection and the reset signal, a modulated light source has been used. Blue light is an optimal excitation signal, being absorbed in the first micrometers of silicon just like an alpha particle. The three signal parameters of such a LED are fully controllable: intensity $A$, duration of pulse $t$ and period $T$, and the parameters $A$ and $t$ were adjusted to simulate exactly the $64 \mathrm{fC}$ of an $\alpha$ particle. In addition, we get control onto the arrival time of the signal, as the diode pulse is triggered by the acquisition cycle of the chip itself. This allows to measure the maximal counting rate $T$, or to generate artificial pile-ups, and also to investigate in detail all the sources of dead time (for incoming particles impinging close to the reset pulse or within the compensating stages). These tests (Fig8) show that the AlphaRad chip is able to run up to $300 \mathrm{kHz}$ for pulsed diode signals simulating the charge deposition of 
alpha particles. This high detection frequency may be understood as a (theoretical) upper detection limit of $1.2 \mathrm{MBq} / \mathrm{cm}^{2}$.

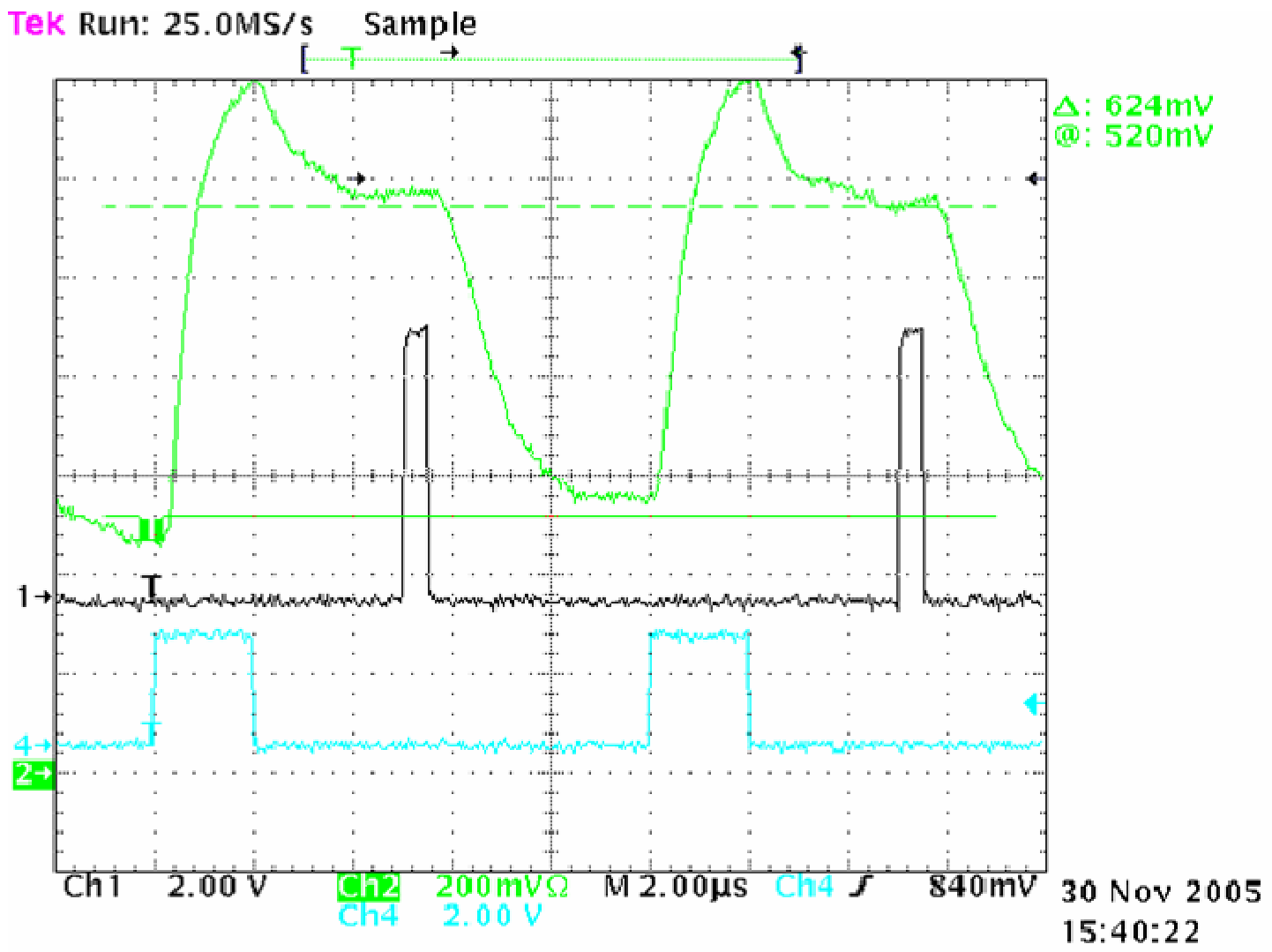

Fig 8: Oscillogram for diode pulses generated close to the reset signal.

\section{Conclusion and perspectives}

We have designed and tested the first demonstrator of a new kind of electronic device for alpha counting, with a $1 / 4 \mathrm{~cm}^{2}$ sensitive area integrated on a VLSI chip, allowing room temperature operation and very simple readout. The measured performances are a nearly $100 \%$ efficiency for $5 \mathrm{MeV}$ alpha particles and a high counting rate (up to $300 \mathrm{kHz}$ or $1.2 \mathrm{MBq} / \mathrm{cm}^{2}$ ). Together with the 
chip's complete transparency to gamma radiation, these features point towards promising applications in neutron dosimetry or monitoring of fast neutrons beams.

This circuit is protected by a European Patent [8].

The next demonstrator (in up-to-date technology) will focus on radiation hardness issues, and it will be designed to test the limits of this concept of large area detection by thermal diffusion for CMOS technologies without epi-layer.

\section{Acknowledgements}

This work was supported in part financially by a special grant from the Scientific Council of the University Louis Pasteur of Strasbourg. We are definetly indebted to our brilliant student H.Mounawar for his hard work in designing the chip in a very short time. We wish to recognize several members of the former LEPSI group of Strasbourg, especially B.Casadei for his early work on this project, C.Colledani and J.D.Berst for their valuable expertise and kind help in the latest stages of the chip design. We acknowledge J.P.Engel for his everyday support in software questions, as well as Prof. Y.Hu and Prof. D.Mathiot for careful reading of the manuscript. 


\section{References}

[1] European Council Directive 97/43/EURATOM, Journal Officiel des Communautés Européennes, June 30, 1997.

[2] 2001/928/EURATOM, Jou.Off.C.E. December 28, 2001.

[3] R. Turchetta, D.Husson et al., A monolithic active pixel sensor for charged particles tracking using standard VLSI CMOS technology, Nucl. Instr. Meth. A458 (2001) 677.

[4] A.Nachab, D.Husson, A.Nourreddine \&al., First measurement of 222Rn activity with a CMOS active pixel system, Nucl. Instr. Meth. B225 (2004) 418422.

[5] D.Husson, Device simulation of a CMOS pixel detector for minimum ionising particle trackin, Nucl. Instr. Meth. A461 (2001) 511.

[6] G.Deptuch, New generation of monolithic active pixel sensors for charged particles detection, PHD Thesis n 4091, ULP Strasbourg, 2002.

[7] J. P. Biersack and J. F. Ziegler. SRIM-2003 codes. http://www.srim.org.

[8] D.Husson and A.Nourreddine, EP 03 360124.6, 28/10/2003. 